\title{
EMBEDDINGS WITH CODIMENSION TWO OF SPHERES IN SPHERES AND $H$-COBORDISMS OF $S^{1} \times S^{3}$
}

\author{
BY JULIUS L. SHANESON
}

Communicated by Richard Palais, April 26, 1968

1. Embeddings with codimension two of spheres in spheres. Let $S^{i}$ denote the standard $i$-sphere with the usual smooth structure, and write $S^{i} \subseteq S^{i+j}$ for the standard inclusion of $S^{i}$ into $S^{i+j}$ on the first $(i+1)$ coordinates.

TheOREM 1.1. Let $\phi: S^{3} \rightarrow S^{5}$ be a smooth embedding. Then the following are equivalent:

(1) $S^{5}-\phi S^{3}$ has the homotopy type of a circle; and

(2) there is a diffeomorphism $f: S^{5} \rightarrow S^{5}$, isotopic to the identity, such that fo is the standard inclusion of $S^{3}$ in $S^{5}$.

That (2) implies (1) is easy. Note that (2) is stronger than the usual unknotting criterion that there be an orientation preserving diffeomorphism of $S^{5}$ throwing $\phi S^{3}$ on to the image of $S^{3}$ under the standard inclusion $S^{3} \subseteq S^{5}$. J. Levine [1] has proven theorems analogous to 1.1 for embeddings of $S^{n}$ in $S^{n+2}, n \geqq 4$, but with (2) replaced by this weaker criterion. This difference is explained by Theorem 1.3 below.

To prove Theorem 1.1, we use the next result.

THEOREM 1.2. Let $M^{5}$ be a smooth manifold of the same homotopy type as $S^{1} \times S^{4}$. Then $M$ is diffeomorphic to $S^{1} \times S^{4}$.

This theorem is proved in the author's thesis and will appear in print with a proof elsewhere. Assuming 1.2, let $\phi$ be as in the statement of 1.1, and suppose that $\phi$ satisfies condition (1). Let $\eta: S^{3} \times D^{2} \rightarrow$ $S^{5}$ be a smooth embedding such that $\eta(x, 0)=\phi(x) ; \eta$ exists because $\pi_{2}(\mathrm{SO}(2))=0$. Let $W$ be the closure of the complement of Image $\eta$. Let $M$ be obtained by surgery using $\eta$; i.e. $M=W \cup_{\eta} D^{4} \times S^{1}$, the disjoint union with $(x, y)$ and $\eta(x, y)$ identified for $(x, y)$ in $S^{3} \times S^{1}$. There is a homotopy equivalence $\alpha: D^{4} \times S^{1} \rightarrow W$ such that $\alpha / S^{3} \times S^{1}$ $=\eta / S^{3} \times S^{1}$. This is not hard to see using the fact that $W$ has the homotopy type of a circle. Hence there is a map

$$
\alpha \cup i d: D^{4} \times S^{1} \cup_{i d} D^{4} \times S^{1} \rightarrow W \cup{ }_{\eta} D^{4} \times S^{1}=M .
$$

It is easy to see that this map is a homotopy equivalence. Moreover, $D^{4} \times S^{1} \cup_{i d} D^{4} \times S^{1}=S^{4} \times S^{1}$. If $x$ is in $D^{4}$ and $y$ in $S^{1}$, let $(x, y)_{2}$ denote 
the corresponding point in the second $D^{4} \times S^{1}$ in the above decomposition of $S^{4} \times S^{1}$. Also, let $\beta: D^{4} \times S^{1} \rightarrow M$ be the inclusion of the second summand.

By Theorem 1.2, there is a diffeomorphism $g: S^{4} \times S^{1} \rightarrow M$. After composing $g$ with $i d \times(-i d): S^{4} \times S^{1} \rightarrow S^{4} \times S^{1}$ if necessary, we can assume after changing $g$ by an ambient isotopy that $g(0, x)_{2}=\beta(0, x)$ for all $x$ in $S^{1}$. Then by the tubular neighborhood theorem it follows after another ambient isotopy that we can actually choose $g$ so that $g(y, x)_{2}=\beta(\mu(x) y, x)$, where $\mu: S^{1} \rightarrow O(4)$. It follows that $g$ restricts to a diffeomorphism $k: D^{4} \times S^{1} \rightarrow W$ such that $k(y, x)=\eta(\mu(x) y, x)$ for $y$ in $S^{3}$ and $x$ in $S^{1}$. (This follows because g must agree with identifications.) Let $h=g \bar{\mu}$, where $\bar{\mu}(y, x)=\left(\mu(x)^{-1} y, x\right)$ for $(y, x)$ in $D^{4} \times S^{1}$. Then $h: D^{4} \times S^{1} \rightarrow W$ is a diffeomorphism such that $h / S^{3} \times S^{1}$ $=\eta / S^{3} \times S^{1}$. Using $h$ and $\eta$, it is now not hard to see that $\phi$ extends to a smooth embedding of $D^{4}$ in $S^{5}$. Namely, define $\phi_{1}: S^{3} \times I \cup D^{4} \rightarrow S^{5}$ as follows, where the domain is the disjoint union of the two summands with $(x, 1)$ and $x$ identified for $x$ in $S^{3}$ : Choose $z$ in $S^{1}$ and let $\phi_{1}(x, t)$ $=\eta(x, t z)$ for $x$ in $S^{3}$ and $\phi_{1}(y)=h(y, z)$ for $y$ in $D^{4}$. Since the domain of $\phi_{1}$ is diffeomorphic to $D^{4}$ via a map extending the inclusion $S^{3} \times 0$ $=S^{3}=\partial D^{4} \subset D^{4}$, this provides the required extension. It follows by a standard result (see [2]) that $\phi$ is ambient isotopic to the standard inclusion, i.e. that (2) in the statement of 1.1 holds.

By generalizing part of the preceding argument slightly and making one or two standard observations, one can prove the first part of the next theorem.

THEOREM 1.3. Let $\phi$ and $\mu$ be two smooth embeddings of $S^{n}$ in $S^{n+2}$ which are unknotted. Let $M_{\phi}$ and $M_{\mu}$ be obtained by surgery using $\phi$ and $\mu$ respectively. Then $M_{\phi}$ and $M_{\mu}$ are well-defined up to diffeomorphism and depend only upon the isotopy class of $\phi$ and of $\mu$, respectively. $M_{\phi}$ and $M_{\mu}$ are diffeomorphic if and only if $\phi$ and $\mu$ are isotopic. Moreover, $M_{\phi}=S^{1} \times \Sigma_{\phi}^{n+1}$, where $\Sigma_{\phi}$ is a homotopy sphere which is well-defined up to diffeomorphism in terms of the isotopy class of $\phi$ if $n \geqq 4$. Given $\Sigma^{n+1}, n \geqq 3$, there is $\phi$ such that $M_{\phi}=S^{1} \times \Sigma$.

To prove that $M_{\phi}=S^{1} \times \Sigma$, we can first suppose after an isotopy that $\phi\left(S^{n}\right)$ is the standard $S^{n}$ in $S^{n+2}$. Let $S^{n} \times D^{2} C S^{n+2}$ be the standard tubular neighborhood of $S^{n}$. Then $\phi \times i d: S^{n} \times D^{2} \rightarrow S^{n} \times D^{2} \subseteq S^{n+2}$, and so we have $M_{\phi}=D^{n+1} \times S^{1} \cup_{\phi \times i d} D^{n+1} \times S^{1}=\Sigma^{n+1} \times S^{1}$, where $\Sigma=D^{n+1} \cup_{\phi} D^{n+1}$. Conversely, if $n \geq 4$, every $\Sigma^{n+1}$ is a twisted sphere $D^{n+1} \cup_{\eta} D^{n+1}, \eta$ a diffeomorphism of $S^{n}$ with itself. It follows from what has been said already that if $\phi$ is the composite of $\eta$ and the standard inclusion of $S^{n}$ in $S^{n+2}, M_{\phi}=S^{1} \times \Sigma$. For $n=3$, we are in the 
case covered by 1.1. That $\Sigma_{\phi}$ is well-defined for $n \geq 4$ follows from standard arguments.

\section{2. $H$-cobordisms of $S^{1} \times S^{3}$.}

THEOREM 2.1. Let $\left(W, \partial_{-} W, \partial_{+} W\right)$ be an h-cobordism such that $\partial_{-} W$ and $\partial_{+} W$ are both diffeomorphic to $S^{1} \times S^{3}$. Then there is a diffeomorphism $h: \partial_{-} W \times I \rightarrow W$ such that $h(x, 0)=x$ for $x$ in $\partial_{-} W$.

For simplicity, we identify $\partial_{-} W=S^{1} \times S^{3}$ and choose a diffeomorphism $\beta: S^{1} \times S^{3} \rightarrow \partial_{+} W$. It is not hard to see that there is a homotopy equivalence $f: S^{1} \times S^{3} \times I \rightarrow W$ which carries $S^{1} \times S^{3} \times 1$ to $\partial_{+} W$ and such that $f(x, y, 0)=(x, y)$ for $x$ in $S^{1}$ and $y$ in $S^{3}$. Every homotopy equivalence of $S^{1} \times S^{3}$ into itself is homotopic to a map of the form $(x, y) \mapsto( \pm x, F(x, y))$, and maps of this form extend over $S^{1} \times D^{4}$ by radial extension. Hence $f$ extends to a map

o: $S^{1} \times D^{4} \cup S^{1} \times S^{3} \times I \cup S^{1} \times D^{4} \rightarrow S^{1} \times D^{4} \cup_{i d} W \cup_{\beta} S^{1} \times D^{4}$.

Let $(x, y)_{i}, i=1$ or 2 , denote a point in either the first or last summand of either of the above two manifolds. In the domain of $g$, for example, $(x, y)_{1}$ is identified with $(x, y, 0)$ for $x$ in $S^{1}$ and $y$ in $S^{3}$, and in the range $\beta(x, y)$ is identified with $(x, y)_{2}$. It is not hard to see that $g$ is a homotopy equivalence and that the domain of $g$ is just $S^{1} \times S^{4}$. Hence by Theorem 1.2 there is a diffeomorphism

$$
k: S^{1} \times D^{4} \cup S^{1} \times S^{3} \times I \cup S^{1} \times D^{4} \rightarrow S^{1} \times D^{4} \cup_{i d} W \cup_{\beta} S^{1} \times D^{4} \text {. }
$$

After composing $k$ with $(-i d) \times i d: S^{1} \times S^{4} \rightarrow S^{1} \times S^{4}$ if necessary and then applying the tubular neighborhood theorem, we can suppose after changing $k$ by an isotopy that $k(x, y)_{1}=\left(x, \mu_{1}(x) y\right)_{1}$ and $k(x, y)_{2}$ $=\left(x, \mu_{2}(x) y\right)_{2}$, where $\mu_{i}$ are maps of $S^{1}$ into $O(4)$. Hence $k$ restricts to a diffeomorphism $h_{1}: S^{1} \times S^{3} \times I \rightarrow W$ such that $h_{1}(x, y, 0)$ $=\left(x, \mu_{1}(x) y\right) \in \partial_{-} W$. Let $h(x, y, t)=h_{1}\left(x, \mu_{1}(x)^{-1} y\right)$. Then $h$ is the required diffeomorphism.

\section{BiBLIOGRAPHY}

1. J. Levine, Unknotting spheres in codimension twa, Topology 4 (1965), 9-16.

2. R. Palais, Extending diffeomorphisms, Proc. Amer. Math. Soc. 11 (1960), 274277.

3. J. L. Shaneson, Ph.D. Thesis, University of Chicago, Chicago, Ill., 1968.

University of Chicago 